\title{
On the Architecture and Design of Multi-Wavelength Passive Optical Arrayed Waveguide Grating Routers
}

\author{
Ali Hammadi \\ Electronics Engineering Department, Public Authority of Applied education and Training (PAAET), Kuwait \\ Email:aa.hammadi@paaet.edu.kw
}

\begin{abstract}
Fiber to the premises (FTTP) technology has become an attractive solution in the last two decades to overcome many limitations appeared with the wireless and copper based technologies in access network. Most of FTTP deployments are based on Passive Optical Network (PON) technology. PONs have become so popular in the optics technology because of its growing reputation in not only providing high speed connectivity but also because of its immunity to EM interference, high security, long distance reach, low cost and low energy consumption. Arrayed Waveguide Grating (AWGs) are passive optical devices that have been widely deployed and implemented in FTTP and many other applications. AWGs can serve as multiplexers, de-multiplexers, filters, routers, and as add/drop devices in many communication systems and optical based applications. In this paper, the AWG PON device is analyzed and simulated using Rsoft simulation tool for the design of multi wavelength optical passive multiplexer and de-multiplexer.
\end{abstract}

Keywords - Passive Optical Network (PON), Arrayed Waveguide Grating (AWG), Fiber to the Premises (FTTP)

\section{INTRODUCTION}

Arrayed Waveguide Grating (AWG) devices have gained so much popularity in the field of optical technology and have been considered for the design of many communication systems and applications [1]. AWGs are found so attractive because of their growing reputation in providing high speed and secure connectivity in the access network. They have been widely deployed and implemented in WDM application especially for providing high speed fiber connectivity to the home, office or the premises (FTTx) [1]. PON Devices such as the AWGRs have shown a radical transformation in communication not only because of the high bit rate that can be delivered but also because of its immunity to EM interference, high security, low cost, low insertion loss, long distance reach and its light weight [2].

AWG based technology makes use of the fact that wavelengths don't interfere with each other while propagating in the same medium or in adjacent channels. AWGs are used to multiplex several channels that have different wavelengths and put them onto a single fiber optic. On the other side AWG is also designed to demuliplex and retrieve individual channels with their different wavelengths at the receiving side on the optical network, where these channels can be connected to different devices and used for several applications like video, voice and data applications.
Recently AWGs are introduced in many WDM technologies and applications. AWGs are found so attractive in the fiber to the premises access network implementation to replace high cost and high power consuming active switches and routers [2]. AWGs are also found in many data center designs to mainly address major concerns raised about the power consumption of IT devices and its impact on global warming in the first place and on the electricity bill in the second place [3]. Such data center architecture designs are promoted and proposed in [4][5][6][7][8][9][10].

In this work, the AWG device is studied and simulated using Rsoft to stand on its functionality and principle of operation for multiplexing and demultiplexing number of wavelengths simultaneously [11]. In Section II, the main components of the simulated AWG device is described. Section III presents the parameters and results obtained from the Rsoft simulation tool for 1:8 mux/demux AWG router. Finally, in Section IV, the paper is concluded.

\section{COMPONENTS OF THE AWG}

AWG device as depicted in Figure 1 consists of two Free Propagation Regions (FPR) star couplers, one at the input and another one at the output. The two FPR are connected with arrayed waveguides with different lengths. The difference of the length $(\Delta \mathrm{L})$ of the arrays is defined by: 

ISSN : 2248-9622, Vol. 7, Issue 6, (Part -1) June 2017, pp.95-99

$$
\Delta \mathrm{L}=\frac{\mathrm{m} \lambda}{\mathrm{n}}
$$

Where $m$ is the order of the array, $n$ is the index of refraction, and $\lambda$ is the center operation wavelength.

Input and output ports are ports connecting the first and second FPRs respectively. In the modeled device shown in Figure 1, one input and eight outputs are set for the full arrayed waveguide. Figure 2 and Figure 3 present the input and the output star couplers respectively.

\section{PARAMETERS AND RESULTS}

The parameters used for the design as recommended by the Rsoft tutorial manual [11] are defined in Table. I.

Table I. WDM AWG Layout

\begin{tabular}{|l|l|}
\hline Number of arrayed waveguides & 40 \\
\hline Center operating wavelength & $1.5 \mu$ \\
\hline Arrays index of refraction & 1.45 \\
\hline Number of input ports & 1 \\
\hline Number of output ports & 8 \\
\hline Wavelength channels & 8 \\
\hline Channel spacing & 0.0016 \\
\hline
\end{tabular}

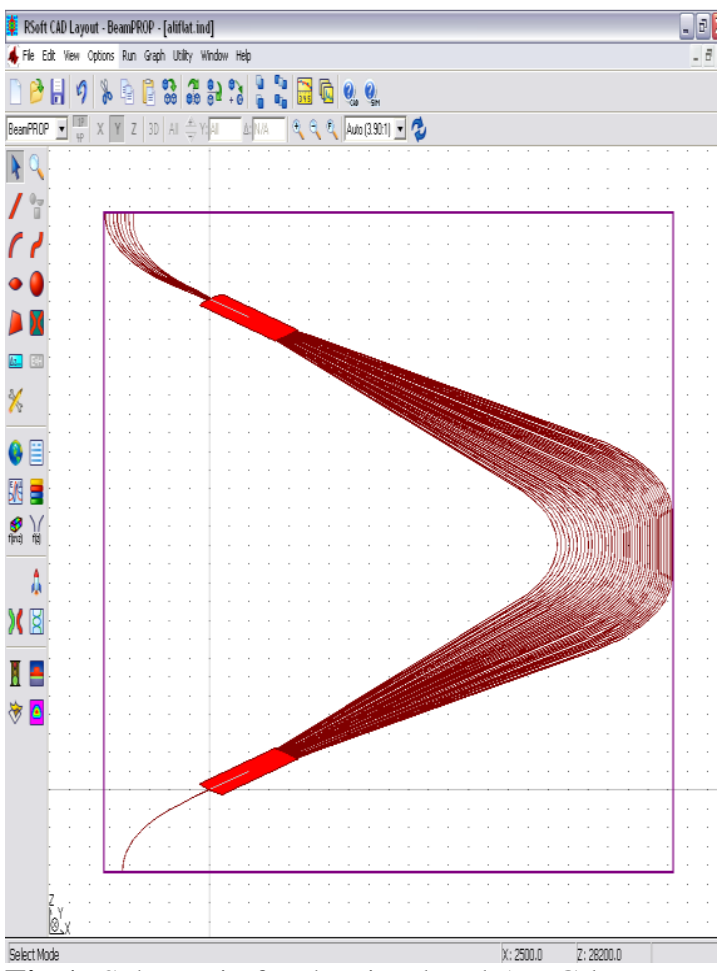

Fig.1: Schematic for the simulated AWG layout

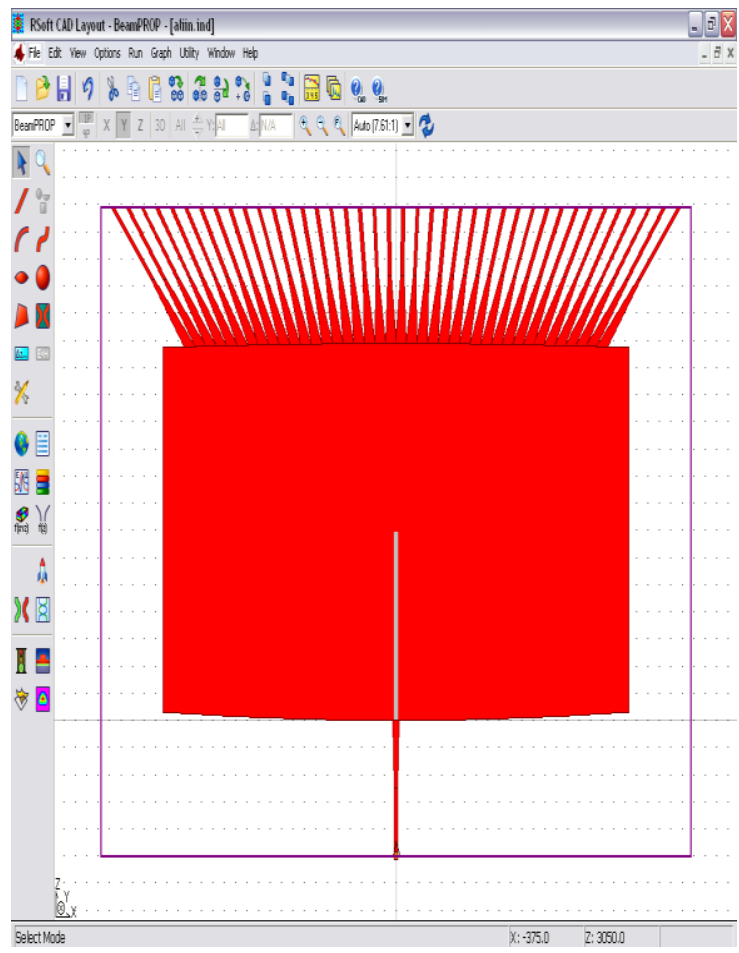

Fig.2: Input star coupler

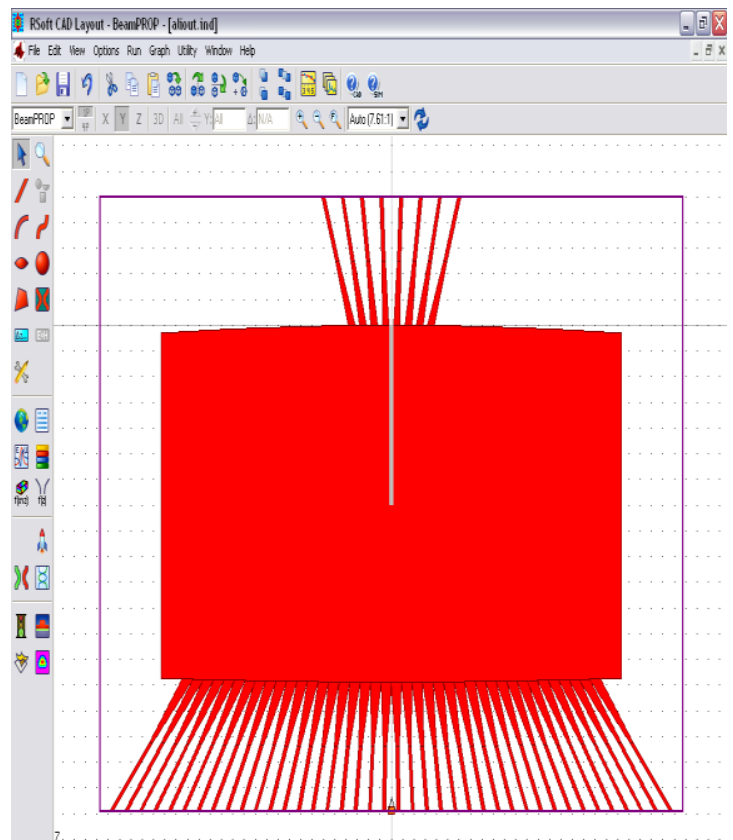

Fig.3: Output star coupler

Figure 4 shows a snapshot from the Rsoft simulation tool for the parameters set as in Table.1 for the design of 1:8 mux/demux AWG router. Figure 5 shows the eigth wavelengths multiplexed at an input port 1 , centered at wavelength $1550 \mathrm{~nm}$ with channel spacing equal to 0.0016. while Figure 6 depicts the demutiplexed channels received at the 8 inputs when the AWG acts as a demiltiplexer. All results are verified with the results described in the Rsoft tutorial manual given in [11]. 


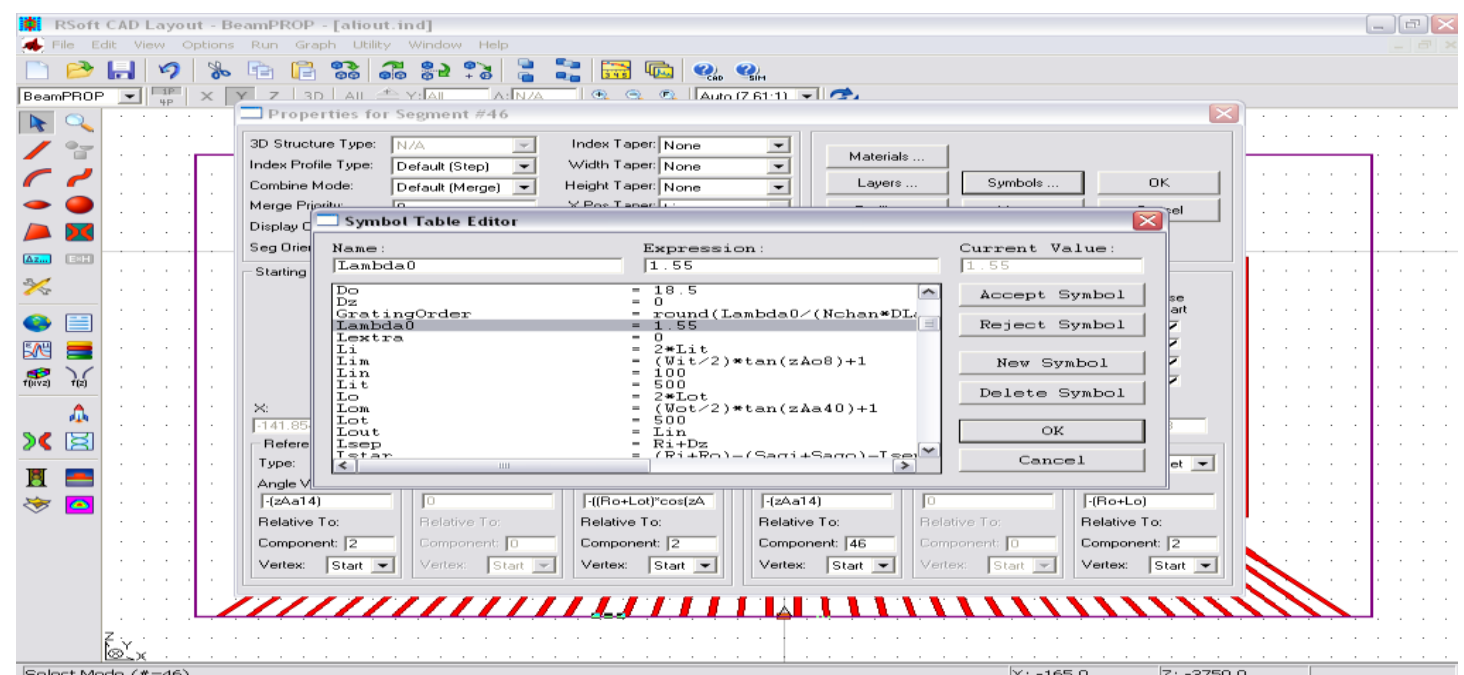

Fig.4: Simulation input parameters



BeamPROP Simulation of AWG WDM Router (Input Port \#1)

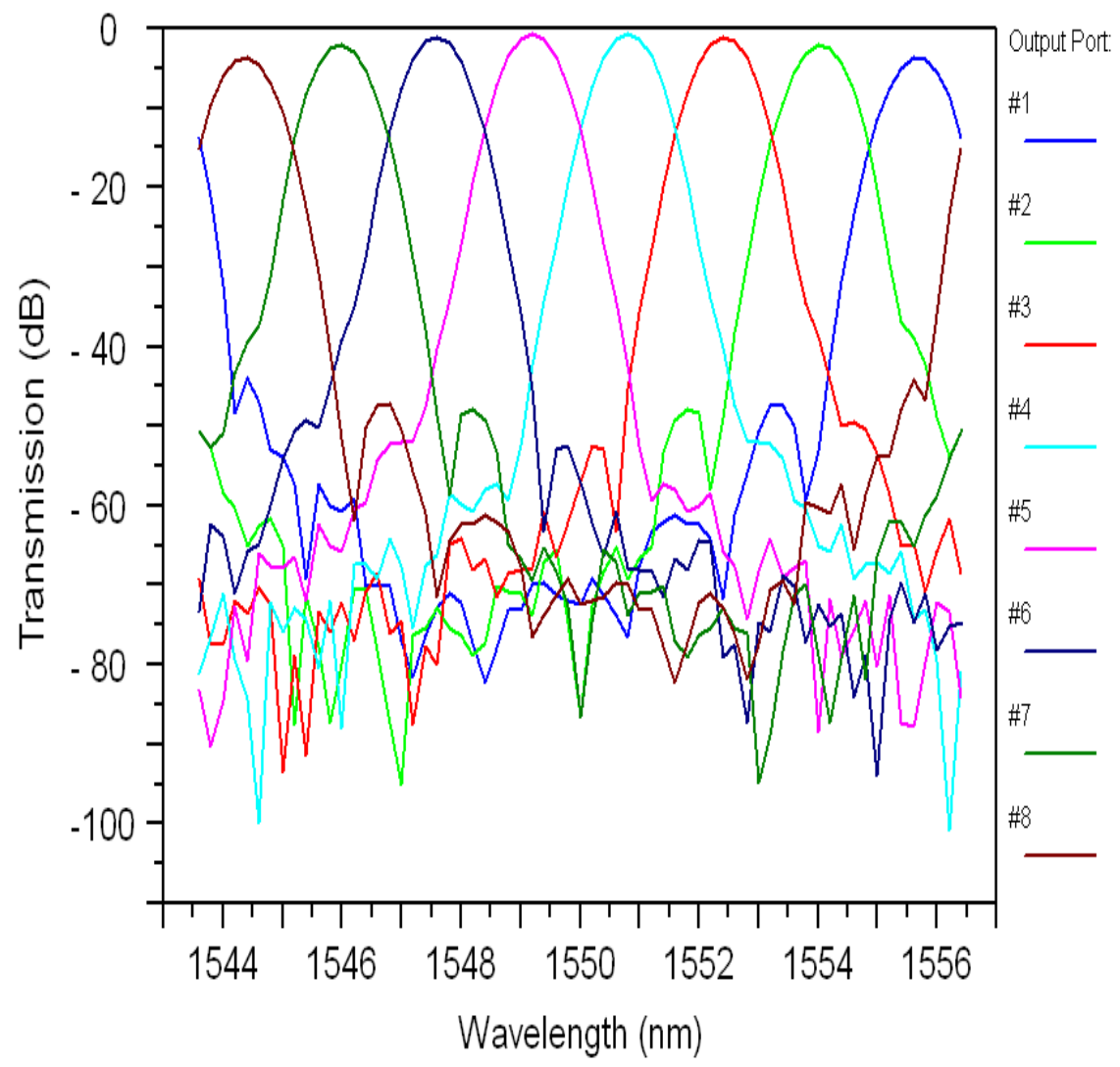

Fig.5: Multiplexed wavelengths at one input port 

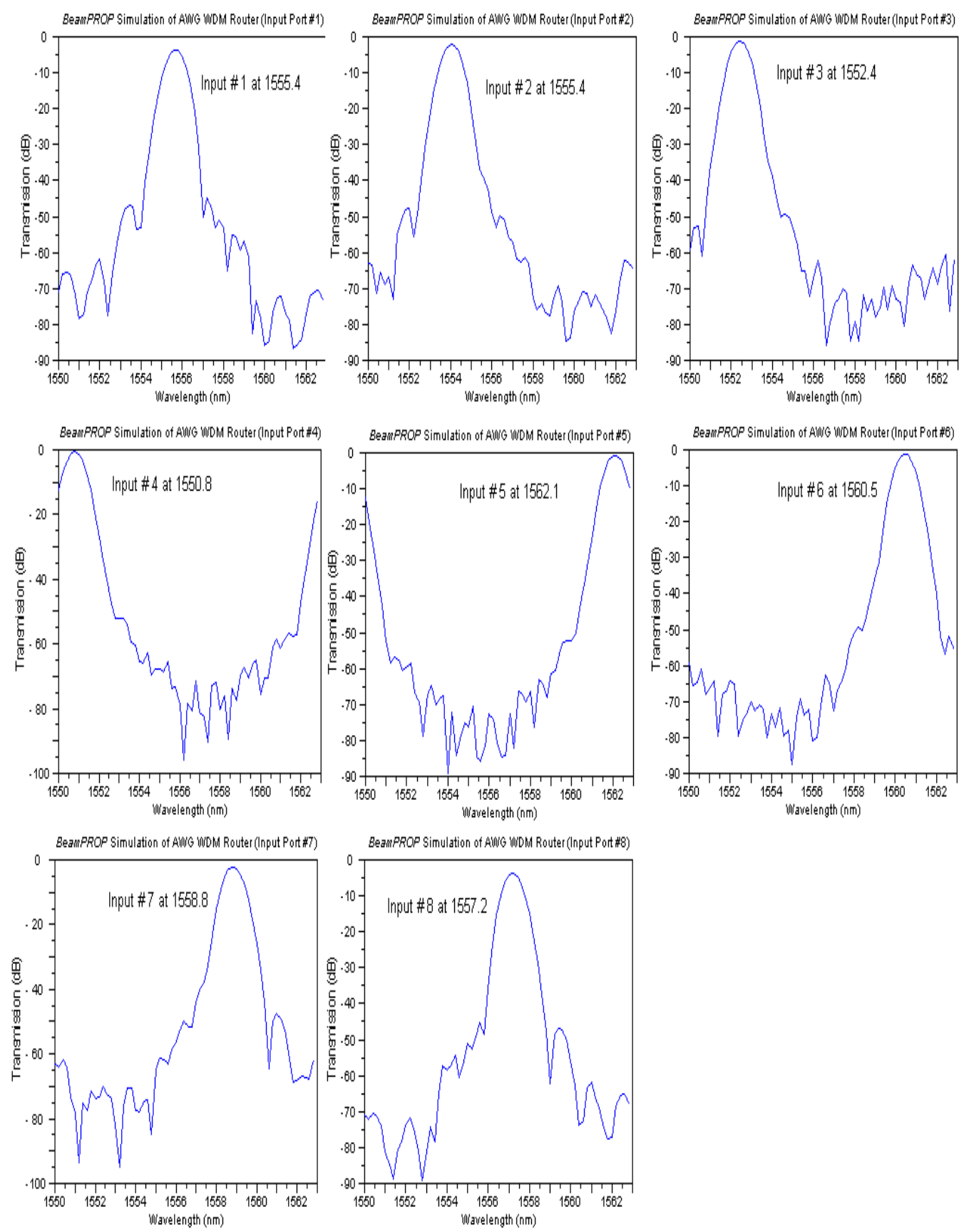

Fig.6: demultiplexed wavelengths at the 8 different inputs of the AWG router

\section{CONCLUSIONS}

AWGs have shown great performance in fiber access networks, data centers, and many other applications and technologies. AWG PONs have resolved many issues appeared in current data centre designs such as high cost and high power consumption resulting from the large number of active switches needed to interconnect almost 1 million servers. PONs have shown great capability to overcome the problems of switch oversubscription along with unbalanced traffic in many networks where PON architectures and protocols have historically been optimized to deal with these problems and handle bursty traffic efficiently. In this work, the AWG router is covered and simulated with the very well-known tool Rsoft simulator to stand on the functionality and principle of operation of the optical passive AWG. The work has shown the results of multiplexing and demultiplexing 8 wavelengths channels simultaneously. 


\section{REFERENCES}

[1] J. C. Palais, Fiber optic communications: Prentice Hall, 1988.

[2] G. Keiser, FTTX concepts and applications vol. 91: John Wiley \& Sons, 2006.

[3] A. Hammadi and L. Mhamdi, "Review: A survey on architectures and energy efficiency in Data Centre Networks," Computer Communication. vol. 40, pp. 1-21, 2014".

[4] Hammadi, T. E. H. El-Gorashi, and J.M.H. Elmirghani, "High Performance AWGR PONs in Data Centre Networks," IEEE International Conference on Transparent Optical Networks, Hungary, 2015.

[5] Hammadi, T. E. H. El-Gorashi, and J.M.H. Elmirghani, "Energy-Efficient SoftwareDefined AWGR-Based PON Data Centre Network" IEEE International Conference on Transparent Optical Network, Italy, 2016

[6] Hammadi, Musa Mohammad, T. E. H. ElGorashi, and J.M.H. Elmirghani, "Resource Provisioning for AWGR-Based PON Cloud Data Centre Network" IEEE 21st European Conference on Network and Optical Communications (NOC), Portugal, 2016.

[7] Hammadi, A., El-Gorashi, T.E.H., Musa, M.O.I. and Elmirghani, J.M.H. (2016) ServerCentric PON Data Center Architecture. 18th International Conference on Transparent Optical Networks, Trento, 10-14 July 2016, 14.

[8] Wang, K., Zhao, L., Gu, H., Yu, X., Wu, G. and Cai, J. (2015) ADON: A Scalable AWGBased Topology for Datacenter Optical Network, Optical and Quantum Electronics, 47 , 2541-2554. https://doi.org/10.1007/s11082-015-0136-z

[9] Elmirghani, J., EL-Gorashi, T. and Hammadi, A. (2016) Passive Optical-Based Data Center Networks. Patent No. WO2016083812 A1.

[10] Hammadi, A. (2017) Mathematical Optimization Modelling for Fast-Switched and Delay Minimized Scheduling for IntraCell Communication in an AWGR-Based PON Data Center. Int. J. Communications, Network and System Sciences, 10, 13-29

[11] Rsoft Design Group, Inc "BeamProp AWG Utility manuel" 